\title{
ARTICLES
}

\section{Engineering and Characterization of Functional Human Microvessels in Immunodeficient Mice}

\author{
Jacques E. Nör, Martin C. Peters, Joan B. Christensen, Michelle M. Sutorik, \\ Stephanie Linn, Mohamed K. Khan, Christina L. Addison, David J. Mooney, and \\ Peter J. Polverini
}

Department of Cariology, Restorative Sciences, and Endodontics (JEN), Department of Oral Medicine/Pathology/
Oncology (MMS, CLA, PJP), and Department of Biologic and Materials Sciences (DJM), School of Dentistry;
Department of Microbiology and Immunology (JBC), Department of Radiation Oncology (SL, MKK), School of
Medicine; Department of Biomedical Engineering (MCP, DJM), School of Engineering, University of Michigan, Ann Arbor, Michigan

SUMMARY: Current model systems used to investigate angiogenesis in vivo rely on the interpretation of results obtained with nonhuman endothelial cells. Recent advances in tissue engineering and molecular biology suggest the possibility of engineering human microvessels in vivo. Here we show that human dermal microvascular endothelial cells (HDMEC) transplanted into severe combined immunodeficient (SCID) mice on biodegradable polymer matrices differentiate into functional human microvessels that anastomose with the mouse vasculature. HDMEC were stably transduced with Flag epitope or alkaline phosphatase to confirm the human origin of the microvessels. Endothelial cells appeared dispersed throughout the sponge 1 day after transplantation, became organized into empty tubular structures by Day 5, and differentiated into functional microvessels within 7 to 10 days. Human microvessels in SCID mice expressed the physiological markers of angiogenesis: CD31, CD34, vascular cellular adhesion molecule 1 (VCAM-1), and intercellular adhesion molecule 1 (ICAM-1). Human endothelial cells became invested by perivascular smooth muscle $\alpha$-actin-expressing mouse cells 21 days after implantation. This model was used previously to demonstrate that overexpression of the antiapoptotic protein Bcl-2 in HDMEC enhances neovascularization, and that apoptotic disruption of tumor microvessels is associated with apoptosis of surrounding tumor cells. The proposed SCID mouse model of human angiogenesis is ideally suited for the study of the physiology of microvessel development, pathologic neovascular responses such as tumor angiogenesis, and for the development and investigation of strategies designed to enhance the neovascularization of engineered human tissues and organs. (Lab Invest 2001, 81:453-463).

$$
T^{\text {had }}
$$
he development of strategies for treatment of angiogenesis-dependent diseases relies on the understanding of their pathogenesis at the cellular and molecular level and the verification of these mechanisms in appropriate in vivo model systems. The classical assays of angiogenesis include the iris and avascular cornea of rodent eye (Gimbrone et al, 1974; Muthukkaruppan and Auerbach, 1979), the chick chorioallantoic membrane (Auerbach et al, 1974), the hamster cheek pouch (Klintworth, 1973), and the dorsal skin and air sac (Folkman et al, 1971). In recent years techniques involving the subcutaneous implan-

Received July 20, 2000.

This work was supported in part by the National Institutes of Health Grants HL39926, CA64416, and DE13161 (PJP); by CAPES Grant 2889/92-3 (JEN); and by a Whitaker Foundation fellowship (MCP). CLA is a research fellow of the National Cancer Institute of Canada.

Address reprint requests to: Dr. Peter J. Polverini, University of Minnesota School of Dentistry, 15-209 Moos Tower, 515 Delaware St. SE, Minneapolis, Minnesota, 55455.E-mail: neovas@tc.umn.edu tation of sterile porous matrices (sponges) have been introduced, and substances to be studied are either injected directly into the sponge or incorporated into slow-release devices (Andrade et al, 1987). Furthermore, naturally occurring and chemically modified matrices such as Matrigel have been used for the study of angiogenesis in vivo (Grant et al, 1993). Although these model systems have certainly proven to be useful for the study of the angiogenic process, they rely on the extrapolation of results obtained in nonhuman tissues or cells for the understanding of the pathogenesis of human diseases.

Recent advances in tissue engineering have improved our ability to generate new tissues through the transplantation of cells into well-defined, biodegradable matrices (Kim and Mooney, 1998). These matrices function as temporary scaffolds that maintain transplanted cells in a defined space and therefore guide new tissue growth and organization. Highly porous biodegradable sponges fabricated from polyL-lactic acid (PLLA) and poly-glycolic acid (PGA) are 
commonly used as temporary scaffolds for tissue engineering (Putnam and Mooney, 1996). The use of biodegradable matrices in severe combined immunodeficient (SCID) mice made it possible to transplant human endothelial cells into a murine host to study the development of human microvessels and the effect of overexpression of the antiapoptotic protein $\mathrm{Bcl}-2$ on human angiogenesis (Nör et al, 1999). Human dermal microvascular endothelial cells (HDMEC) transplanted in PLLA sponges into SCID mice organize and differentiate into functional microvessels that anastomose with mouse vasculature and become functional human microvessels containing mouse blood cells (Nör et al, 1999, 2000).

Here we describe the development and characterization of this novel bioassay, the SCID mouse model of human angiogenesis, that can be used to examine both physiological and pathologic human angiogenesis in mice. We show that the microvessels in polymer implants are lined with human endothelial cells, are invested by murine smooth muscle cells, express specific markers of angiogenesis, and transport murine blood cells. Potential applications of this model system include the study of how overexpression of genes in endothelial cells affect neovascularization; the testing of the effect of antiangiogenesis therapeutic strategies in tumor growth; and the evaluation of how the survival of endothelial cells can be genetically manipulated to enhance the vascularization and blood supply to engineered tissues.

\section{Results}

\section{Human Endothelial Cells Transplanted into SCID Mice Form Human Microvessels}

Highly porous PLLA sponges were used as scaffolds to transplant human endothelial cells into SCID mice (Fig. 1a). The sponges used had an average pore diameter of $250 \mu \mathrm{m}$ (Fig. 1b), which allowed for easy penetration and entrapment of endothelial cells seeded in a 1:1 mix of culture medium and Matrigel (Fig. 1, c and d). Matrigel functions as an immediate source of growth factors and nutrients for the endothelial cells, as well as a means to maintain these cells in the sponge during the transplantation process. The percentage of cells that are actually retained in the sponge is $68 \%$ ( $\pm 4 \%$ ). Therefore, when $1 \times 10^{6}$ cells
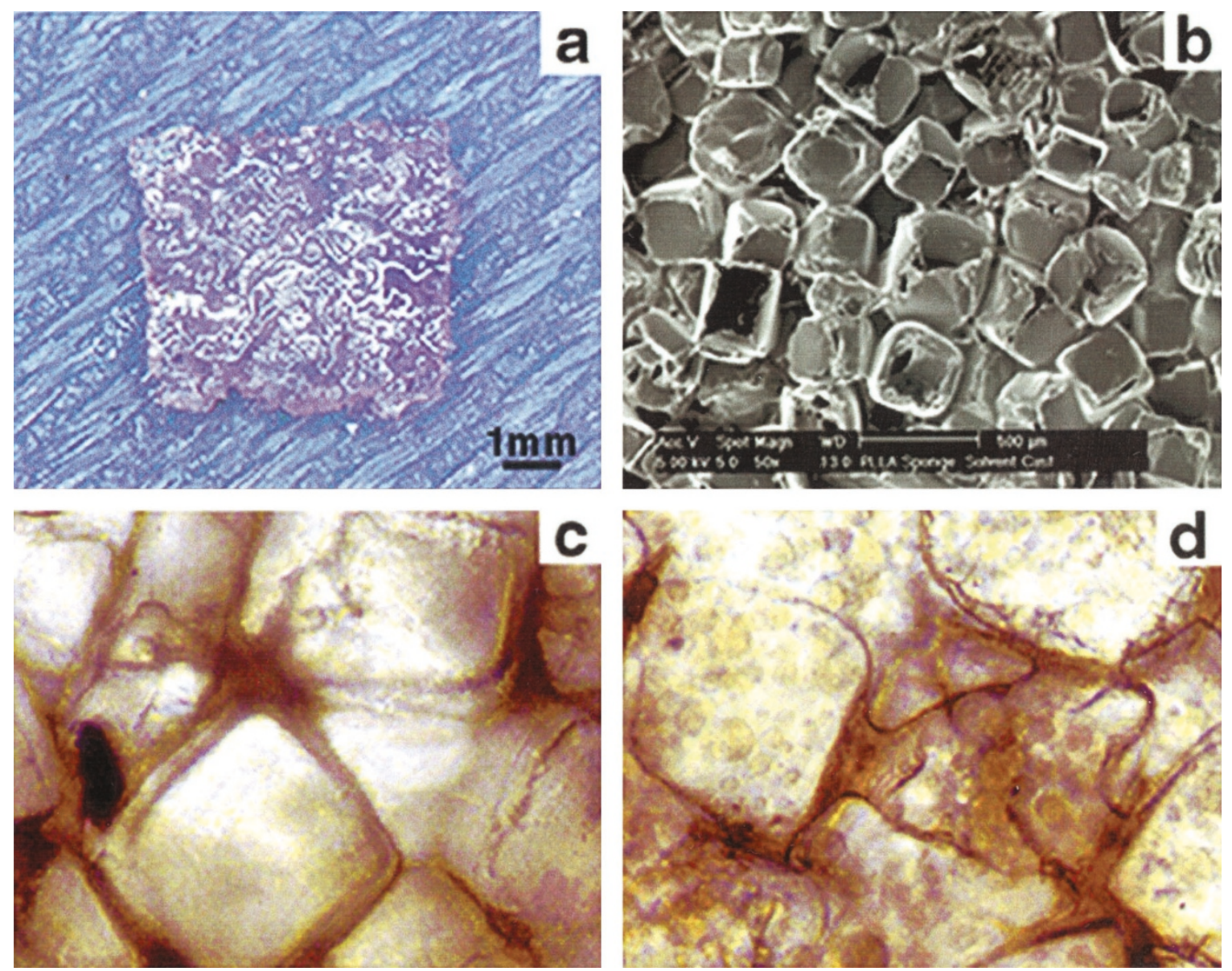

Figure 1.

Highly porous poly-L-lactic acid (PLLA) matrices provide a scaffold for transplantation of endothelial cells into severe combined immunodeficient (SCID) mice. a, PLLA matrices were cut to $6 \times 6 \times 1-\mathrm{mm}$ sponges with an average pore diameter of $250 \mu \mathrm{m}$, as seen by scanning electron microscopy, original magnification, $\times 90$ (b), or by phase microscopy, original magnification, $\times 200$ (c). d, Human dermal microvascular endothelial cells (HDMEC) were resuspended in a 1:1 EGM-MV and Matrigel mixture and seeded at $1 \times 10^{6}$ cells/sponge (original magnification, $\times 200$ ). HDMEC appear dispersed throughout the sponge (original magnification, $\left.\times 200\right)$. 
are seeded into a $6 \times 6 \times 1$-mm sponge, on average $6.8 \times 10^{5}\left( \pm 4.3 \times 10^{4}\right)$ cells are retained in the sponge and transplanted into the mice.

Sponges seeded with human endothelial cells were implanted bilaterally in the dorsal subcutaneous tissue of SCID mice. At the time of implant retrieval (up to 28 days), no signs of infection were detected and inflammation was minimal. Implants maintained in mice for at least 5 days became encapsulated by a fibrous connective tissue (Fig. 2a) that was permeated by mouse blood vessels (Fig. 2b). Macroscopically the implants appear as highly vascularized tissues (Fig. 2c), and histologic examination shows an abundance of highly organized microvessels that are immunoreactive with human CD31 and CD34 (Fig. 2d), two endothelial cell-specific markers (Miettinen et al, 1994; Vermeulen et al, 1996).

To determine the origin of the cells that populate the microvessels inside the implants we used several approaches. We performed immunostaining with antihuman CD31 (Fig. 3a) and antihuman CD34 (Fig. 3b) antibodies. These antibodies only reacted with endothelial cells from microvessels localized inside the implant and not with the ones present in the surrounding fascia and connective tissue. To confirm that the microvessels in the implant were populated with the transplanted human endothelial cells, we used retroviral infection to transduce HDMEC and to generate stable clonal populations of cells expressing either the artificial Flag epitope or the alkaline phosphatase gene. These two cell populations were implanted in independent sponges in SCID mice and after 14 days the implants were retrieved and stained for either Flag (Fig. 3c) or alkaline phosphatase (Fig. 3d). In both cases, only microvessels inside the implants showed positive staining for the markers used, demonstrating that these microvessels were populated by transplanted human endothelial cells and not by host cells at this time point. The microvessels present in the connective tissue that surrounds the implants were invariably negative for immunostaining with the markers used in this study.

To further characterize these microvessels, we examined the expression of vascular cellular adhesion molecule 1 (VCAM-1) and intercellular adhesion molecule 1 (ICAM-1), two mediators of endothelial cell-cell interactions and regulators of angiogenic responses (Koch et al, 1995; Polverini, 1996). VCAM-1 is an inducible endothelial cellular adhesion molecule that is expressed in stimulated HDMEC (Swerlick et al, 1992), and ICAM-1 is a regulated ligand for lymphocyteendothelial cell adhesion (Dustin and Springer, 1988). We found that at 7 days approximately $60 \%$ of the microvessels in the implants expressed these adhesion molecules, whereas at 14 days this proportion was reduced to approximately $25 \%$ (Fig. 4a). The
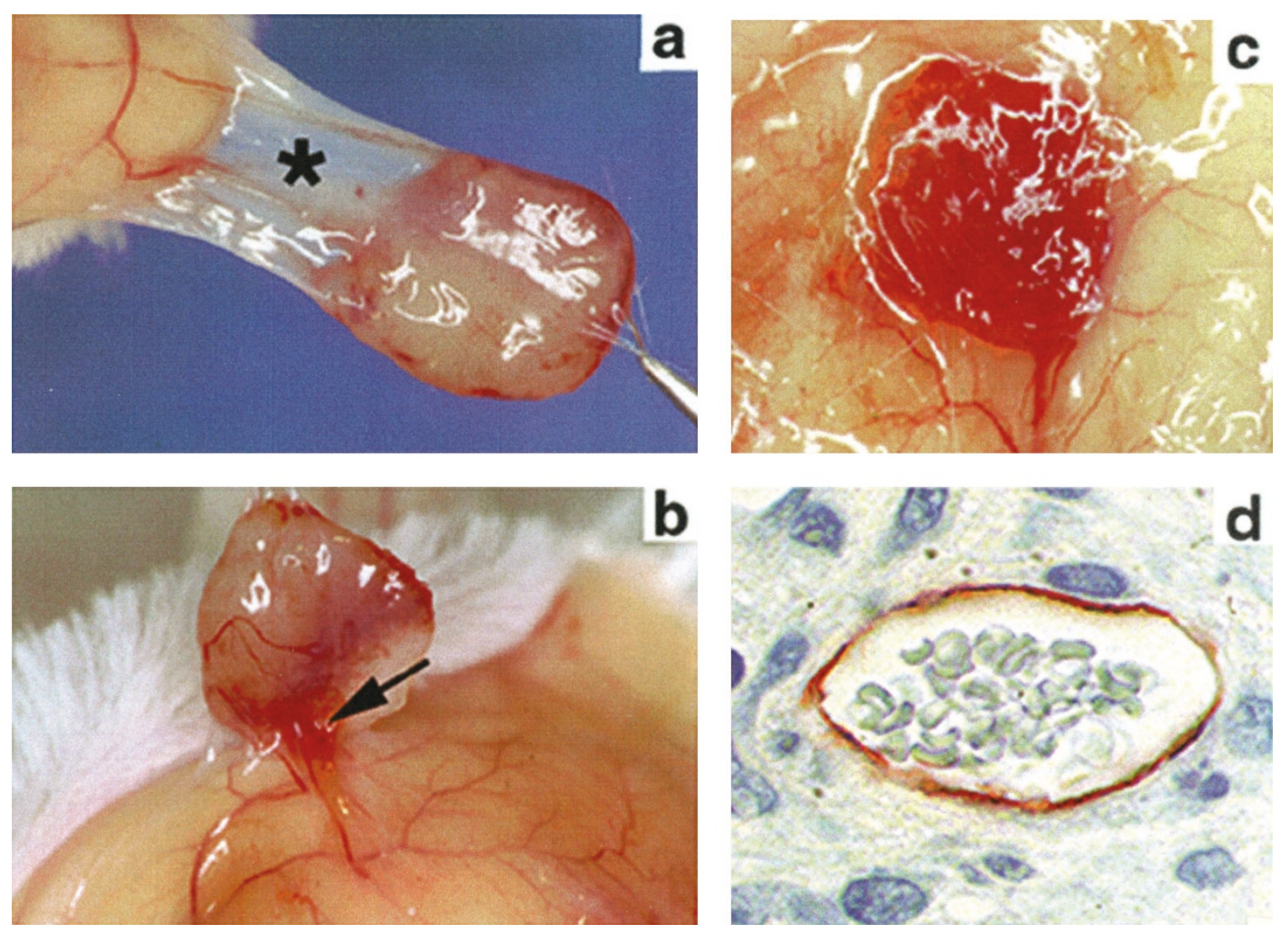

Figure 2.

HDMEC organize into functional microvessels that anastomose with mouse vasculature. a, A fibrous fascia (*) involves the sponge implants 14 days after implantation. b, Mouse blood vessels penetrate the surrounding fascia, and c, the implant has the appearance of a highly vascularized tissue. $d$, Photomicrograph of a microvessel (original magnification, $\times 1,000$ ) in the implant, stained positively for human CD34 and containing mouse blood cells. 

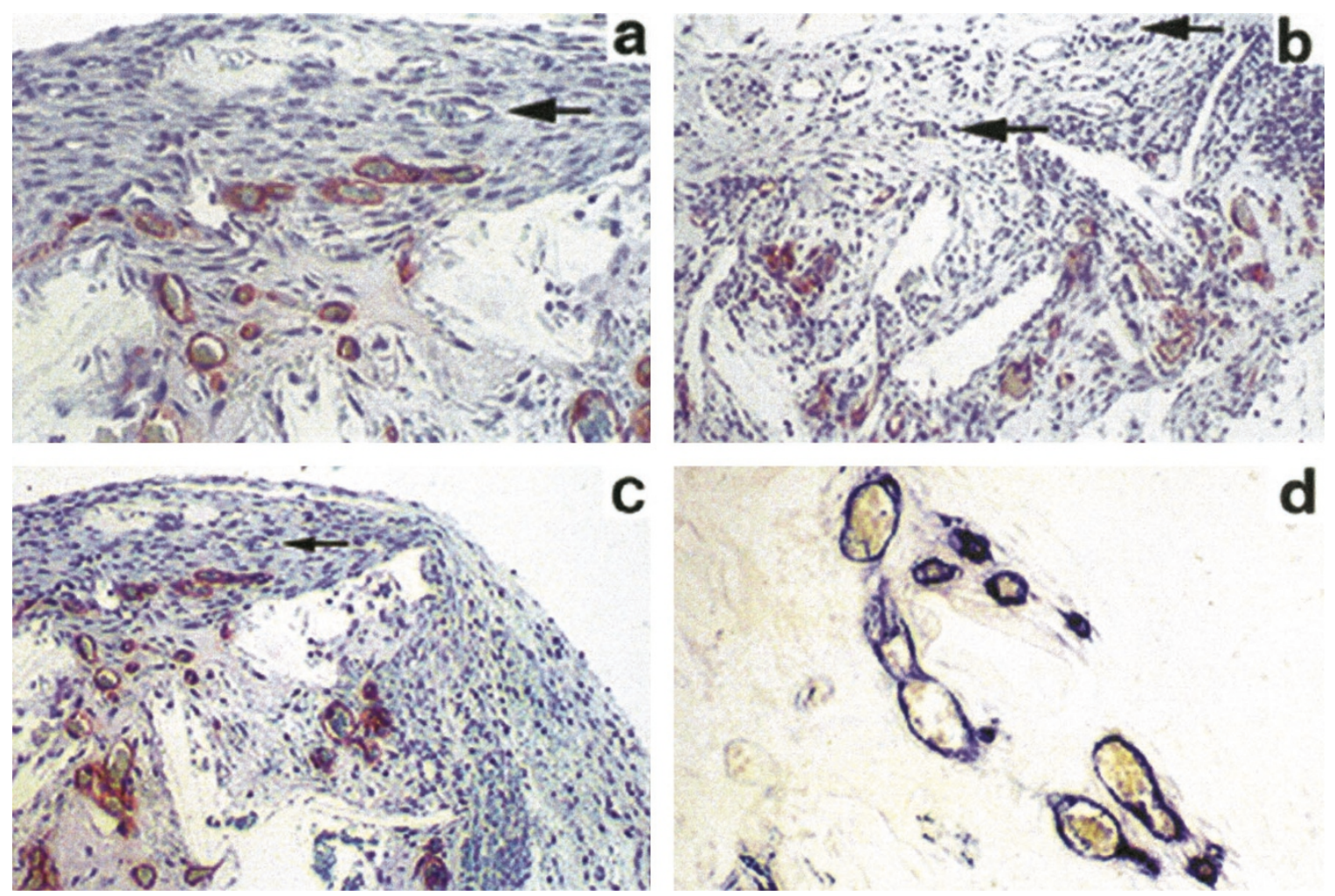

Figure 3.

Microvessels inside the implants are lined with human endothelial cells. Immunostaining with antihuman CD31 (a) or antihuman CD34 (b) antibodies results in positive staining of only microvessels inside the implants. Immunostaining with anti-Flag (c) antibody also results in positive staining of only microvessels inside sponges that were populated with HDMEC stably transduced with this epitope. HDMEC stably transduced with alkaline phosphatase and transplanted into SCID mice develop functional human microvessels containing blood cells (d). Black arrows point to mouse microvessels in the surrounding fibrous fascia or connective tissue that were not stained with the markers used to identify human transplanted endothelial cells. (a and d, original magnification, $\times 200, b$ and $c$, original magnification, $\times 100$ ).

decrease in expression of these two adhesion molecules in microvessels over time can be explained, at least in part, by the fact that their expression levels seem to be reduced in more mature or quiescent blood vessels (Strömblad and Cheresh, 1996).

\section{Engineered Human Microvessels Anastomose with Mouse Vasculature and Differentiate into Functional Microvessels}

To understand the time required for transplanted endothelial cells to organize into functional microvessels, we performed a time course experiment and used CD31 as a marker to identify HDMEC (Fig. 4b). Five days after implantation we found a high number of nonfunctional tubular structures containing empty lumens. Starting on day 7 and thereafter, we observed the presence of murine blood cells in the lumen of these structures, which we termed functional microvessels. From Day 7 to Day 14, we observed a significant drop in the number of nonfunctional microvessels, and at Day 21 they had essentially disappeared. We found that approximately one of five of the tubular structures that developed initially anastomosed with the mouse vasculature to become differentiated into functional microvessels. This suggests that blood flow mediates signals to maintain these microvessels, and that tubular structures that did not anastomose with resident mouse vessels regressed.
Representative histologic fields illustrate the progression from isolated endothelial cells at Day 1 to functional microvessels at Day 14. One day after implantation all endothelial cells were dispersed throughout the sponges (Fig. 5a). At Day 5, tubular structures in stages of development ranging from partially open to complete cylinders could be seen throughout the implants (Fig. 5b). Two days later, cells from the walls of microvessels started to migrate toward the center (Fig. 5c), dividing these tubules into two or three separate lumens (Fig. 5d). At 14 days, the majority of the tubules seen in histologic sections represented functional microvessels containing murine blood cells (Fig. 5e). Interestingly, we observed that some microvessels located at the periphery of the implants contained both cells that were positive and others that were clearly negative for immunostaining with antihuman CD34 antibody (Fig. 5f). We believe that these areas are composed of both human and mouse cells, and we called them chimeric microvessels. These may be areas of anastomosis, where the human and the mouse vasculature connect, allowing for the engineered human microvessels to become functional blood-carrying microvessels.

To determine whether the human microvessels become invested by mouse perivascular smooth muscle cells, we performed immunostaining with anti- $\alpha$ smooth muscle actin antibody. Seven days after im- 
a

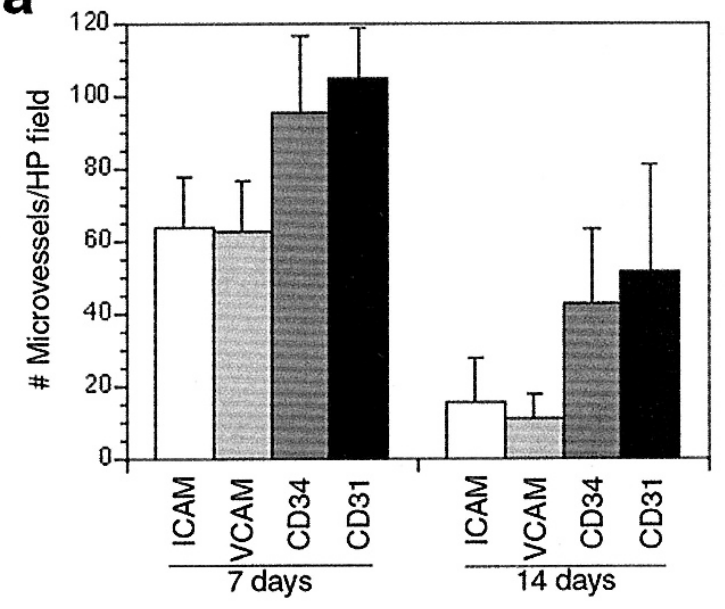

b

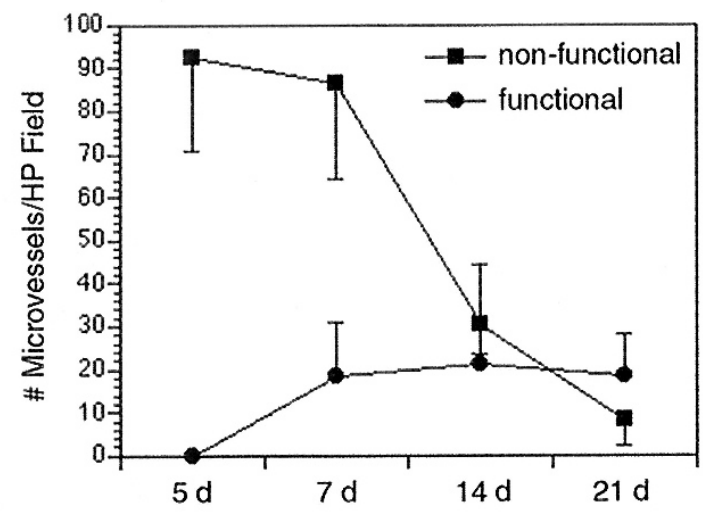

Figure 4.

Engineered microvessels express a panel of markers of physiologic angiogenesis and become functional over time. a, Sections of sponges retrieved 7 or 14 days after implantation were immunostained using antihuman vascular cellular adhesion molecule 1 (VCAM-1), and intercellular adhesion molecule 1 (ICAM-1), CD31, and CD34 monoclonal antibodies. The number of positively stained microvessels was counted in 10 random fields from at least four independent implants per time point at $\times 200$ magnification. $b$, The number of microvessels stained with antihuman CD31 antibody was counted over time. Microvessels were categorized as functional (with blood cells in the lumen) or nonfunctional (without blood cells). CD $31^{+}$microvessels were evaluated in 10 random fields from at least four independent implants per time point at $\times 200$ magnification.
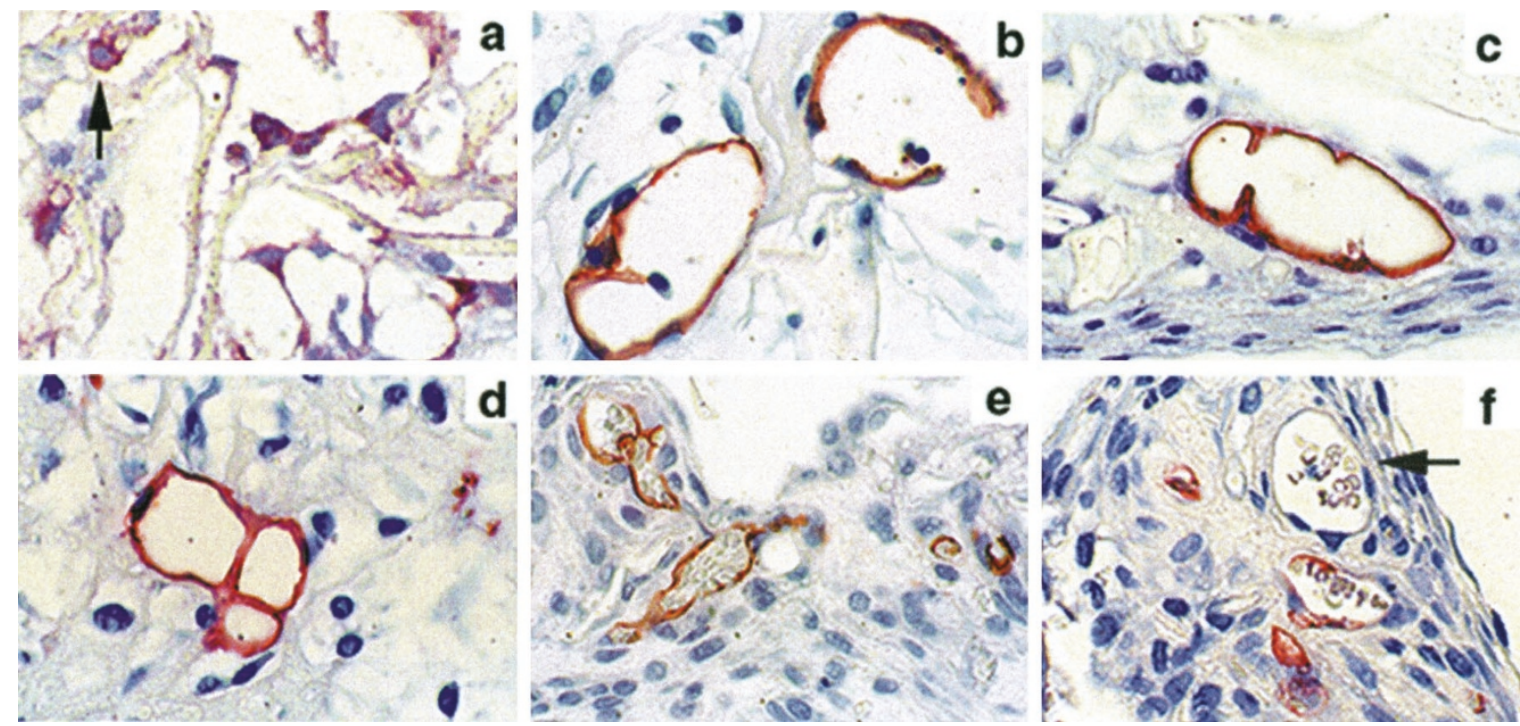

Figure 5.

Development of human microvessels in SCID mice. a and b, Immunostaining with antihuman CD34 antibody was used to identify human endothelial cells. Arrow in Panel A points to a CD34 positive endothelial cell. HDMEC are dispersed throughout the sponge 1 day after implantation (a), and form empty cylinders after 5 days (b). $\mathrm{c}$ and d, Endothelial cells from the walls start to migrate inward at around Days 5 to 7 , dividing the large nonfunctional microvessel into two or three smaller structures. e and f, Fourteen days after implantation, mouse blood cells can be seen in the lumen of most microvessels. f, The arrow points to a mouse microvessel, not stained with antihuman CD34 antibody, located at the periphery of the implant. All photomicrographs are original magnification, $\times 1,000$.

plantation, empty tubular structures lined with endothelial cells could be seen throughout the implants (as described above), but these structures did not show signs of staining for smooth muscle cells (Fig. 6a). At 14 days, a weak staining for $\alpha$-smooth muscle actin could be observed in a few microvessels (Fig. 6b). However, 3 weeks after implantation most microvessels showed clearly a layer of perivascular $\alpha$-smooth muscle actin-positive mouse cells investing the endothelial cells (Fig. 6c).
Four weeks after implantation some of the HDMEC lining the walls of implant microvessels were apoptotic, as demonstrated by double staining with antiCD34 antibody and TUNEL (Fig. 7, a to c). Interestingly, the total number of microvessels (determined by staining with Von Willebrand factor) inside the implants remained unaltered at this time point (Fig. 7d). We determined that 14 days after implantation the proportion of microvessels lined with human endothelial cells was $90 \%$ to $100 \%$, whereas after 28 days this 

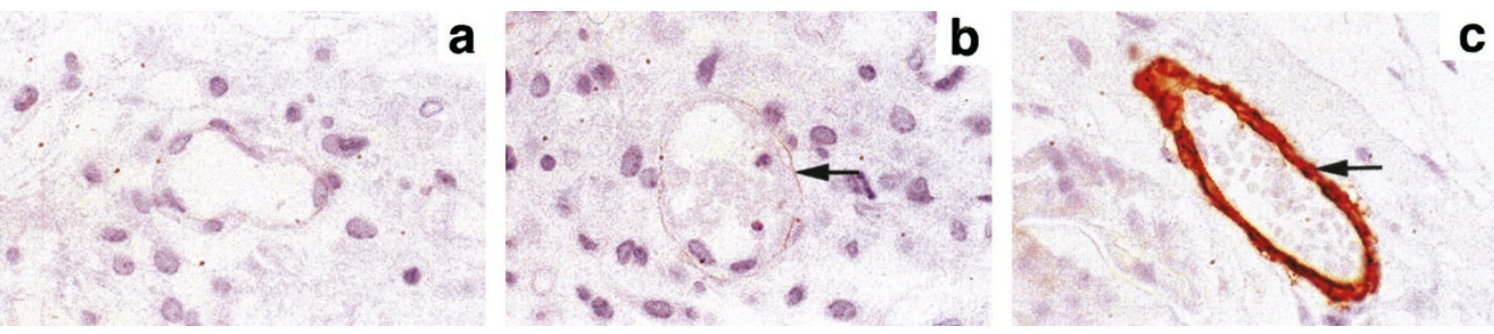

Figure 6.

Perivascular smooth muscle cells invest human microvessels in SCID mice. Immunostaining with anti- $\alpha$-smooth muscle actin antibody was used to identify smooth muscle cells. a, HDMEC form nonfunctional microvessels 7 days after implantation without perivascular smooth muscle cells. b, Fourteen days after implantation a weak staining for $\alpha$-smooth muscle actin can be seen, and c, at 21 days most implant microvessels present an investing layer of $\alpha$-smooth muscle actin-expressing mouse cells. The arrows point to perivascular $\alpha$-smooth muscle actin-expressing mouse cells. All photomicrographs are original magnification, $\times 1,000$.

proportion had decreased to $50 \%$ to $65 \%$ (Fig. $7 d$ ). Taken together, these data suggest that apoptotic HDMEC are substituted by mouse endothelial cells that maintained the integrity of the microvessels and the intraimplant microvascular density.

\section{Applications of the SCID Mouse Model of Human Angiogenesis}

This model system can be used to study several aspects of angiogenesis. The effect of a gene in endothelial cells can be studied by transplanting stable clones of endothelial cells overexpressing, or lacking, this gene. We have previously demonstrated that overexpression of the antiapoptotic protein $\mathrm{Bcl}-2$ in endothelial cells significantly enhances neovascularization of implants (Fig. 8, a and b) (Nör et al, 1999). The relevance of endothelial cells to tumor growth can be seen in Figure 8c. In one sponge we transplanted only oral squamous cell carcinoma cells (OSCC-3) and in the other a mixture of endothelial cells overexpressing Bcl-2 and OSCC-3. After 21 days, the tumors that
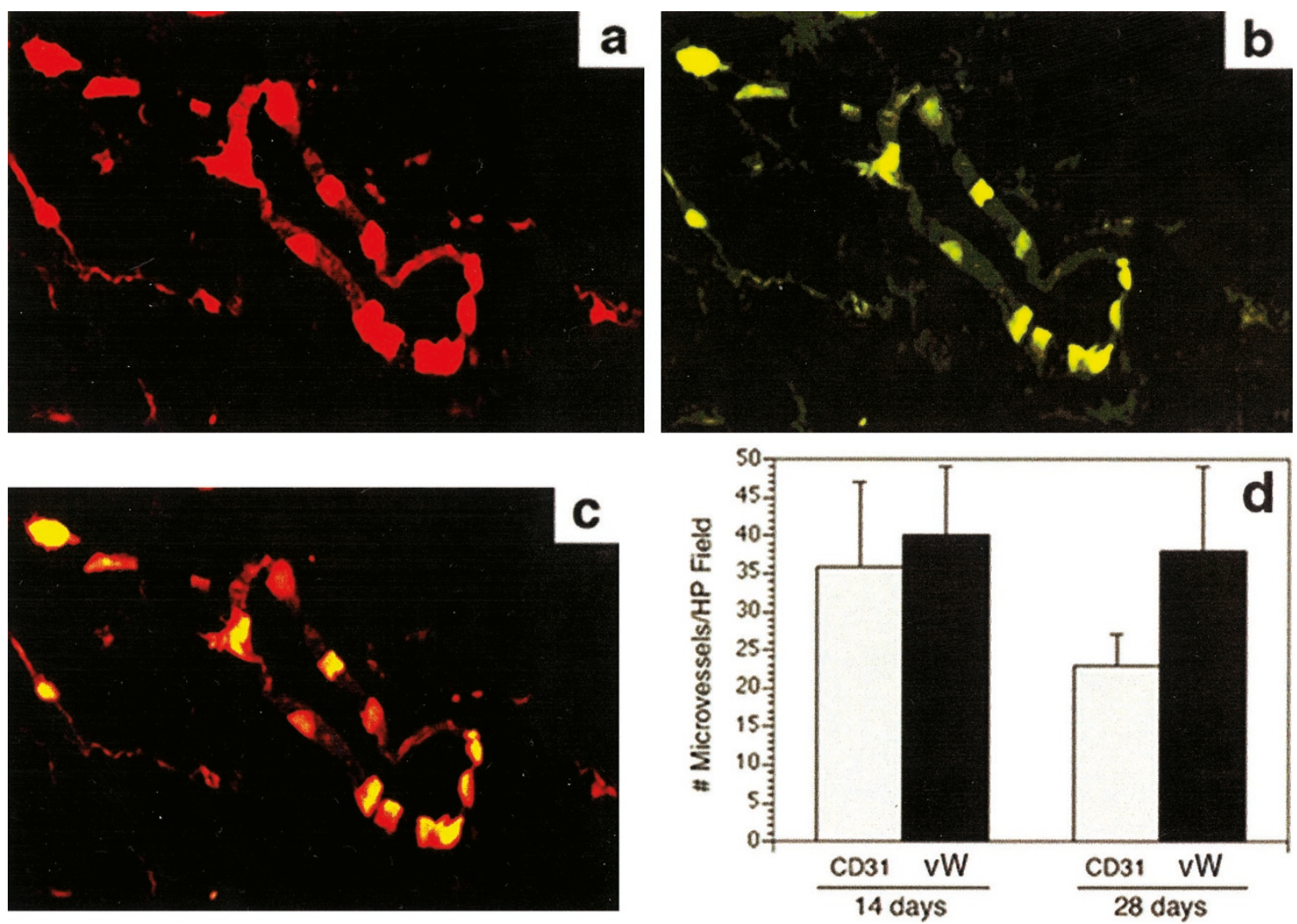

Figure 7.

Apoptosis of microvessel HDMEC 4 weeks after implantation. $a$ and b, Tissue sections were stained for human CD31-Texas Red (a) and in situ TUNEL-fluorescein (b), and analyzed by confocal microscopy. c, The images obtained in panels a and b were overlapped. Apoptotic HDMEC are shown as bright yellow cells. d, The proportion of human vs mouse microvessels over time was determined by immunostaining consecutive tissue sections with either polyclonal antihuman Von Willebrand factor antibody (which cross-reacts with both human and mouse endothelial cells) or antihuman CD31 antibody (a marker for human endothelial cells only). All photomicrographs are original magnification, $\times 1,000$. 
developed from sponges containing both OSCC-3 and endothelial cells were significantly larger than the ones populated with tumor cells alone (Nör et al, 2001). This model system can also be used to study morphological aspects of the human tumor microvasculature with defined endothelial and tumor cell populations (Fig. 8d). Furthermore, one can study the effects of endothelial cell survival on tumor cell survival. Tumor cells surrounding a functional microvessel have the required oxygen and nutrient influx to survive and to proliferate (Fig. 8e). In contrast, tumor cells surrounding an apoptotic microvessel also become apoptotic, as demonstrated by TUNEL assay (Fig. 8f). The findings described here demonstrate the versatility of this model system and suggest its potential use for the study of both physiological and pathologic angiogenesis in vivo.

\section{Discussion}

Despite significant advances in experimental models of angiogenesis, the lack of simple, reliable, reproducible, and quantitative assays still hinders the progress of research in this area (Auerbach et al, 1991). We describe here a novel model system that combines recent advances in tissue engineering with developments in cellular biology and molecular genetics that allow for the study of human angiogenesis in a murine host.

This experimental model can be used to study physiologic angiogenesis. One can use this model system to examine the initial steps that result in the alignment of endothelial cells into tubular structures. We observed that within 5 to 7 days of implantation, cells that were initially spread throughout the sponge organize into empty tubular structures. Therefore, this system is valuable for the study of the function of chemotactic molecules or soluble endothelial-derived factors that mediate their approximation and organization into tubular structures. We have also observed that once these cells have aligned and formed large tubular structures, endothelial cells from the walls start to migrate toward the center of the lumen, dividing the original tubule into two or three. This perhaps recapitulates processes leading to sprouting of microvessels seen during physiologic angiogenesis.

Seven to 14 days after implantation, the majority of the microvessels inside the implants evolve from empty tubular structures to functional blood-carrying microvessels. We have carefully determined that these microvessels were human and not simply invading mouse vasculature inside the implant. We demonstrated that the transplanted human endothelial cells lining the microvessels developed inside the implants by performing immunostaining with anti-Flag antibody in sections of implants containing endothelial cells stably transduced with this artificial epitope. We also performed alkaline phosphatase staining in implants seeded with a clonal population of endothelial cells engineered to overexpress this gene. The transition between empty tubular structures to functional bloodcarrying microvessels implies that the tubules lined with human cells can anastomose with the existing mouse vasculature. Chimeric mouse-human tubules, at the periphery of implants, may represent crosssections of areas in which the engineered human microvessels connect with the mouse vasculature. These areas contained cells that were clearly stained for the markers used to identify human transplanted
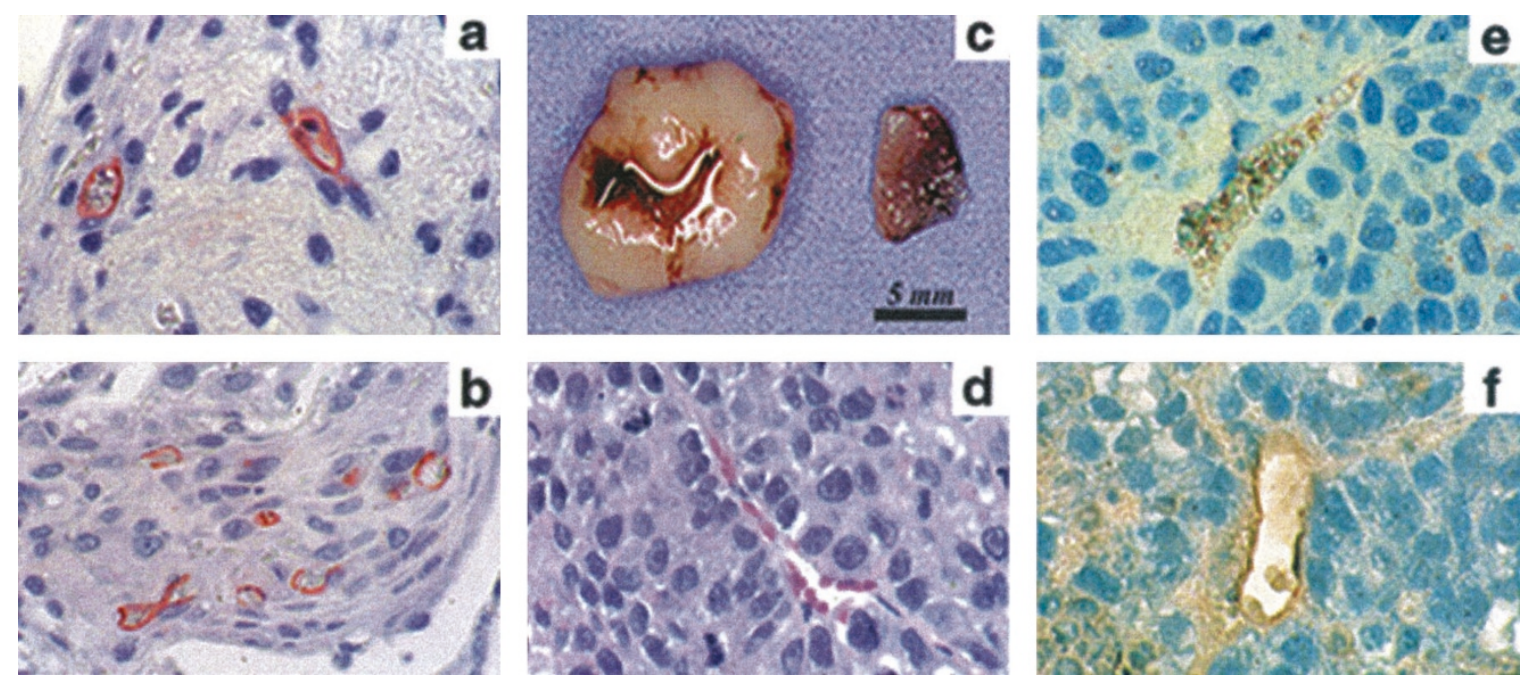

Figure 8.

Potential applications of the SCID mouse model of human angiogenesis. a and b, The effect of the overexpression of endothelial cell genes on neovascularization. A sponge implant seeded with empty vector control HDMEC (a) is less vascularized than an implant containing HDMEC overexpressing the antiapoptotic bcl-2 gene (b) 14 days after transplantation. c, The effect of the expression of an antiapoptotic gene on endothelial cells populating a tumor on tumor growth. The implant on the left was populated with HDMEC-BCl-2 and OSCC-3, whereas the implant on the right was populated with 0SCC-3 only. Both tumors were retrieved from the same mouse 21 days after implantation. $d$, Tumor vasculature with defined populations of tumor and endothelial cells. Hematoxylin and eosin staining of sponge implant seeded with oral squamous cell carcinoma cells (OSCC-3) and HDMEC, 21 days after implantation. e and f, The effect of endothelial cell apoptosis on tumor cell apoptosis. TUNEL-stained sections of implants containing HDMEC-Bcl-2 and OSCC-3 (e) or vector control HDMEC and OSCC-3 (f). All photomicrographs are original magnification, $\times 1,000$ (except Panel $\mathrm{C}, \times 1$ ). 
endothelial cells and other endothelial cells that did not stain for these markers.

To further characterize the engineered microvessels, we examined the expression of two cellular adhesion molecules that mediate physiologic angiogenic responses. We observed that a significant proportion of these microvessels is immunoreactive to antihuman VCAM-1 and ICAM-1 antibodies. The expression of these two markers has been associated with active angiogenesis processes (Polverini, 1996). We found that at later time points, such as 21 days (Fig. 4a) and 28 days (data not shown) after transplantation, the proportion of microvessels expressing these two markers decreased, suggesting that these microvessels are in more mature stages of differentiation. We have also found that 21 days after implantation most microvessels became invested by murine perivascular smooth muscle cells (Fig. 6). Taken together, these data suggest that engineered microvessels lined with untransduced endothelial cells behave similarly to naturally occurring angiogenic microvessels, from both a functional and a genotypic standpoint. In a recent report, Schechner and colleagues (2000) described a methodology for developing human vasculature in SCID mice that requires the preorganization of microvessels in vitro (using collagen and fibronectin) for 20 hours before implantation. The implantation of these constructs containing untransduced HUVEC into SCID mice resulted in thin-walled microvessels after 31 days without the ingrowth of smooth muscle cells. This is in contrast to our findings that demonstrate that the implantation of untransduced HDMEC in biodegradable scaffolds resulted in rapid differentiation of functional microvessels as soon as 7 days after implantation, and that these microvessels are lined by perivascular $\alpha$-smooth muscle actinexpressing mouse cells.

A limitation of our model system is the increasing presence of mouse microvessels in the implants over time, probably attributable to the fact that some HDMEC undergo apoptosis. This phenomenon was more noticeable 28 days after implantation and thereafter, when a significant proportion of the microvessels either contained TUNEL $^{+}$HDMEC or no longer stained for the markers used for the identification of the human endothelial cells. Because of this shortcoming, we believe that our model system is ideally suited for the study of the earlier stages of physiologic or pathologic neovascularization. However, the survival of endothelial cells in implants can be dramatically enhanced by overexpressing antiapoptotic genes in these cells. We have previously demonstrated that $\mathrm{Bcl}-2$ overexpression mediated by retroviral transduction of human endothelial cells enhanced the survival of human microvessels in SCID mice (Nör et al, 1999). This finding was recently corroborated by others (Schechner et al, 2000). Although Bcl-2 overexpression certainly increases intraimplant microvascular density over time, the genetic manipulation of the endothelial cells before implantation may interfere with the ability to understand physiologic human angiogenesis in SCID mice. Therefore, we believe that untransduced endothelial cells should be used whenever the objective of the investigation is to understand mechanisms of physiologic angiogenesis.

An additional application of the model system described here is the study of the effects of angiogenic or antiangiogenic factors secreted by a second population of cells that is cotransplanted with HDMEC in the sponges. We have cotransplanted fibroblasts that overexpress and secrete high levels of thrombospondin-1 (TSP1), or truncated forms of this protein, with endothelial cells and evaluated the effect of this inhibitor of angiogenesis on implant neovascularization (Nör et al, 2000). We showed a significant inhibition of angiogenesis in implants containing HDMEC and fibroblasts overexpressing TSP1, or TSP1truncated forms that retained the antiangiogenic type I repeats, compared with cotransplantation of HDMEC and fibroblasts. The decrease in intraimplant microvascular density was mediated by enhanced apoptosis of endothelial cells lining implant microvessels. These findings demonstrated the usefulness of this model system for studying the effect of secreted antiangiogenic factors in vivo.

One of the current limitations of tissue engineering is the ability to provide sufficient blood supply for tissues and organs in the initial phase after transplantation. The SCID mouse model of human angiogenesis may be used to evaluate the efficacy of delivering locally angiogenic factors. Cotransplantation of cells that overexpress and secrete soluble angiogenic factors may enhance the vascularization of engineered tissues. These cells may serve as short-term delivery devices of angiogenic stimuli to enhance initial vascularization of engineered tissues. Alternatively, the efficacy of modified scaffolds containing angiogenic factors such as VEGF or bFGF for enhancement of tissue neovascularization may also be tested with this model system.

Cotransplantation of human endothelial cells and tumor cells resulted in the development of human tumors populated by human microvessels in SCID mice. Therefore, one can examine the role of angiogenesis in tumor progression in a system that uses defined populations of human tumor cells and endothelial cells. Twenty-one days after transplantation of two different tumor cell lines (OSCC-3 and SLK), we observed the growth of large encapsulated tumors in the sponge implants (Nör et al, 2001). Tumors that developed from these implants were indistinguishable histologically from the respective primary tumors, as determined by two experienced pathologists. We observed that cotransplantation of OSCC-3 and endothelial cells resulted in enhanced tumor volume and weight compared with transplantation of tumor cells alone, underlining the role of angiogenesis in tumor growth (Nör et al, 2001). To further understand the role of pathologic angiogenesis in tumor progression, one can cotransplant tumor cells and endothelial cells genetically modified to overexpress or knockout the expression of specific genes. We cotransplanted endothelial cells overexpressing $\mathrm{Bcl}-2$ together with tumor cells and found that these tumors were signifi- 
cantly larger after 21 days than tumors arising after cotransplantation of empty vector control endothelial cells and tumor cells (Nör et al, 2001). In the same experiment, we found a significantly smaller number of apoptotic microvessels and a concomitant enhancement in intratumoral microvascular density when endothelial cells overexpressing $\mathrm{Bcl}-2$ were used as compared with control endothelial cells. The possibility of defining the cell populations of the angiogenic and tumoral compartments enhances the ability to study the impact of each one of these components on the overall progression of tumors. Furthermore, it allows one to genetically modify these cells, characterize them in vitro, and then study the behavior of these cells in vivo.

The SCID mouse model of human angiogenesis was developed using recent advances in tissue engineering and molecular genetics. This system is simple, reproducible, and can be used to study both physiologic and pathologic angiogenic processes. It allows for the study of human angiogenic processes in a murine host by implanting defined cell populations. We believe that this model system provides an opportunity to enhance our understanding of both physiologic and pathologic human angiogenesis.

\section{Materials and Methods}

\section{Cells}

HDMEC (Clonetics, San Diego, California) were cultured in microvascular endothelial cell growth medium (EGM-MV; Clonetics). HDMEC were transduced with pLAPSN retroviral vector (a gift from D. Miller) and selected with $250 \mu \mathrm{g} / \mathrm{ml}$ G418 sulfate (Mediatech, Herndon, Virginia) in EGM-MV to generate stable clones overexpressing the alkaline phosphatase (AP) protein. The generation of HDMEC-Bcl-2-Flag and HDMEC-LXSN (empty vector control) stable clones was performed as described (Nör et al, 1999). Human oral squamous carcinoma cells (OSCC-3, a gift from M. Lingen) were cultured in Dulbecco's modified Eagle's medium (Gibco BRL, Grand Island, New York) supplemented with $10 \%$ FBS.

\section{Biodegradable Polymer Matrix}

Porous poly-L-lactic acid (PLLA) sponges were fabricated as previously described (Mooney et al, 1997). Briefly, PLLA (Medisorb, Cincinnati, Ohio) was dissolved in chloroform to yield a solution of $5 \%$ polymer $(\mathrm{w} / \mathrm{v})$, and $1.67 \mathrm{ml}$ of this solution was loaded into siliconized glass beakers packed with $2.3 \mathrm{~g}$ of sodium chloride particles. The solvent was allowed to evaporate and the sponges were subsequently immersed for 16 hours in distilled $\mathrm{H}_{2} \mathrm{O}$ to leach the salt and to create an interconnected pore structure in the sponges. The sponges, which had an average pore diameter of 250 $\mu \mathrm{m}$, were cut to $6 \times 6 \times 1 \mathrm{~mm}$ and sterilized by gamma radiation. The day before transplantation, sponges were soaked in 100\% EtOH for 2 hours, washed twice with PBS, and then left overnight in fresh PBS.

\section{Scanning Electron Microscopy}

Dried sponges were attached to sample stands with carbon glue and then gold coated in a sputter coater. Imaging was performed with a Hitachi S-800 field emission scanning electron microscope.

\section{Transplantation of Endothelial Cells}

Just before transplantation, $1 \times 10^{6}$ untransduced HDMEC, HDMEC overexpressing Flag-tagged Bcl-2, HDMEC overexpressing alkaline phosphatase, or empty vector controls were resuspended in $36 \mu \mathrm{l}$ of a 1:1 mixture of EGM-MV and growth factor-reduced Matrigel (Collaborative Biomedical Products, Cambridge, Massachusetts) and allowed to adsorb into the sponges. The effect of endothelial cells on tumor growth was examined by seeding either $0.9 \times 10^{6}$ HDMEC-BCL-Z with $0.1 \times 10^{6}$ OSCC-3 or only $0.1 \times$ $10^{6}$ OSCC-3 in PLLA sponges (Nör et al, 2001). The sponges were incubated for 30 minutes at $37^{\circ} \mathrm{C}$ to allow for the gelation of Matrigel. Male 3- to 4-weekold SCID mice (CB.17.SCID; Taconic, Germantown, New York) were anesthetized with ketamine and xylazine, and then two sponges were implanted subcutaneously in the dorsal region of each mouse. One to 28 days after transplantation, the mice were killed and the implants were retrieved, immediately measured with calipers, and weighed on an electronic balance. The implants were fixed overnight in $10 \%$ buffered formalin at $4^{\circ} \mathrm{C}$, embedded in paraffin, and mounted on Superfrost (Fisher Scientific, Pittsburgh, Pennsylvania) glass slides for histologic examination. The care and treatment of experimental animals was in accordance with the University of Michigan's guidelines.

Immunolocalization of VCAM-1, ICAM-1, CD31, CD34, Flag, Von Willebrand Factor, and $\alpha$-Smooth Muscle Actin

Tissue sections were deparaffinized and antigen retrieval was achieved by pressure cooking (Decloaking Chamber; Biocare Medical, Walnut Creek, California) at $120^{\circ} \mathrm{C}$ for 2 minutes in citrate buffer $(2.94 \mathrm{~g} / \mathrm{l}$ sodium citrate dihydrate, $\mathrm{pH}$ 6.0). For immunostaining of VCAM-1 and ICAM-1, sections were blocked for 5 minutes with $10 \%$ rabbit serum in PBS, then with Sniper (Biocare Medical) for an additional 5 minutes. This was followed by an overnight incubation at $4^{\circ} \mathrm{C}$ with polyclonal goat antihuman VCAM-1 (R\&D Systems, Minneapolis, Minnesota) or polyclonal goat antihuman ICAM-1 antibodies (R\&D Systems) diluted in Sniper at 1:250 and 1:300, respectively. Sections were incubated with appropriate secondary antibodies for 30 minutes, followed by incubation with streptavidinHRP (Biocare Medical). The Dakoark kit (Dako, Carpinteria, California) was used to minimize background in sections that were stained for $\alpha$-smooth muscle actin. A 1:50 dilution of monoclonal mouse anti- $\alpha$-smooth muscle actin (1A4; Dako) was applied for 30 minutes at $37^{\circ} \mathrm{C}$ to the sections. For immunostaining with CD31, CD34, and Von Willebrand factor, tissue sections were blocked with Sniper for 5 minutes, and incubated overnight at $4^{\circ} \mathrm{C}$ with $41 \mu \mathrm{g} / \mathrm{ml}$ monoclonal 
antihuman CD31 (JC/70A; Dako), $10 \mu \mathrm{g} / \mathrm{ml}$ monoclonal antihuman CD34 (QBEnd/10; Lab Vision Corporation, Freemont, California), or $28 \mu \mathrm{g} / \mathrm{ml}$ rabbit antihuman Von Willebrand factor (Dako) diluted in PBS. Sections were incubated with $44 \mu \mathrm{g} / \mathrm{ml}$ anti-Flag M2 monoclonal antibody (Sigma, St. Louis, Missouri) for 1 hour at $37^{\circ} \mathrm{C}$. Sections were incubated with appropriate secondary antibodies for 30 minutes, followed by processing with a Lincoln Label $4^{+}$Detection System (Biocare Medical). The DAB 500 Chromogen System (Biocare Medical) or a solution of $0.014 \mathrm{~g}$ of 3-amino9-ethyl carbazole (Sigma) in $2.5 \mathrm{ml}$ of $\mathrm{N}, \mathrm{N}$ dimethylformamide (Sigma) were used to visualize bound antibodies. The number of stained microvessels was counted blindly in 10 random fields per implant at $\times 200$ magnification. Four or five implants were analyzed per condition and time point.

\section{Double Staining for CD31 and Apoptosis}

Tissue sections were deparaffinized and antigen retrieval was achieved as describe above. Sections were blocked with Sniper for 5 minutes and incubated overnight at $4^{\circ} \mathrm{C}$ with $41 \mu \mathrm{g} / \mathrm{ml}$ monoclonal antihuman CD31 (JC/70A; Dako) diluted in PBS. The sections were incubated with biotinylated antimouse $\lg G$ (Biocare Medical) for 30 minutes, followed by a 30minute incubation with $3 \mu \mathrm{g} / \mathrm{ml}$ avidin-Texas Red conjugate (Vector Laboratories, Burlingame, California) at room temperature. After a 10-minute rinse in PBS, the ApopTag fluorescein in situ apoptosis detection kit (Intergen, Purchase, New York) was used according to manufacturer's instructions. Tissue sections were examined in a confocal microscope (MRC600 CLSM; Bio-Rad, Hercules, California) at $\times 1,000$ magnification.

\section{Staining for Alkaline Phosphatase}

Retrieved sponges were cut into thin slices and fixed in $10 \%$ buffered formalin overnight. After fixation, sponges were washed three times in PBS at room temperature and once in PBS at $65^{\circ} \mathrm{C}$ for 30 minutes to inactivate any endogenous phosphatase activity. This was followed by a wash in AP buffer $(100 \mathrm{~mm}$ TrisHCl, $\mathrm{pH} 8.5,100 \mathrm{~mm} \mathrm{NaCl}, 50 \mathrm{~mm} \mathrm{MgCl}_{2}$ ) for 10 minutes at room temperature. The sponge slices were stained in a solution of $100 \mu \mathrm{g} / \mathrm{ml}$ BCIP (5-bromo-4chloro-3-indolyl-phosphate; Boehringer Mannheim, Indianapolis, Indiana), $0.5 \mathrm{ng} / \mathrm{ml}$ NBT (nitroblue tetrazolium chloride), $0.02 \%$ NP40 (Sigma), and 0.01\% sodium deoxycholate (Sigma) in AP buffer overnight at room temperature in the dark. The reaction was stopped with $20 \mathrm{~mm}$ EDTA in PBS, and the slices were embedded in paraffin for histologic evaluation.

\section{In Situ TUNEL Assay}

In situ TUNEL staining (ApopTag peroxidase in situ detection kit) was used according to manufacturer's instructions to identify apoptotic cells.

\section{Acknowledgements}

The authors thank M. Lingen for the gift of cells, D. Miller for retrovirus vectors and packaging cell lines, C. Edwards for technical support with the confocal microscopy, and the Research Histology and Immunoperoxidase Laboratory at the University of Michigan Comprehensive Cancer Center for the immunohistochemistry work.

\section{References}

Andrade SP, Fan TP, and Lewis GP (1987). Quantitative in vivo studies on angiogenesis in a rat sponge model. $\mathrm{Br} \mathrm{J}$ Exp Pathol 68:755-766.

Auerbach R, Auerbach W, and Polakowski I (1991). Assays for angiogenesis: A review. Pharmacol Ther 51:1-11.

Auerbach R, Kubai L, Knighton D, and Folkman J (1974). A simple procedure for the long-term cultivation of chicken embryos. Dev Biol 41:391-394.

Dustin ML and Springer TA (1988). Lymphocyte functionassociated antigen-1 (LFA-1) interaction with intercellular adhesion molecule-1 (ICAM-1) is one of at least three mechanisms for lymphocyte adhesion to cultured endothelial cells. J Cell Biol 107:321-331.

Gimbrone MA Jr, Cotran RS, Leapman SB, and Folkman J (1974). Tumor growth and neovascularization: An experimental model using the rabbit cornea. J Natl Cancer Inst 52:413-427.

Folkman J, Merler E, Abernathy C, and Williams G (1971). Isolation of a tumor factor responsible or angiogenesis. J Exp Med 133:275-288.

Grant DS, Kleinman HK, Goldberg ID, Bhargava MM, Nickoloff BJ, Kinsella JL, Polverini PJ, and Rosen EM (1993). Scatter factor induces blood vessel formation in vivo. Proc Natl Acad Sci USA 90:1937-1941.

Kim BS and Mooney DJ (1998). Development of biocompatible synthetic extracellular matrices for tissue engineering. Trends Biotechnol 16:224-230.

Klintworth GK (1973). The hamster cheek pouch: An experimental model of corneal vascularization. Am J Pathol 73: 691-710.

Koch AE, Halloran MM, Haskell CJ, Shah MR, and Polverini PJ (1995). Angiogenesis mediated by soluble forms of E-selectin and vascular cell adhesion molecule-1. Nature 376:517-519.

Miettinen M, Lindenmayer AE, and Chaubal A (1994). Endothelial cell markers CD31, CD34, and BNH9 antibody to $\mathrm{H}$ and $Y$-antigens: Evaluation of their specificity and sensitivity in the diagnosis of vascular tumors and comparison with Von Willebrand factor. Mod Pathol 7:82-90.

Mooney DJ, Sano K, Kaufmann PM, Majahod K, Schloo B, Vacanti JP, and Langer R (1997). Long-term engraftment of hepatocytes transplanted on biodegradable polymer sponges. J Biomed Mater Res 37:413-420.

Muthukkaruppan V and Auerbach R (1979). Angiogenesis in the mouse cornea. Science 205:1416-1418.

Nör JE, Christensen J, Liu J, Peters M, Mooney DJ, Strieter $\mathrm{RM}$, and Polverini PJ (2001). Up-regulation of Bcl-2 in microvascular endothelial cells enhances intratumoral angiogenesis and accelerates tumor growth. Cancer Res 61:21832188. 
Nör JE, Christensen J, Mooney DJ, and Polverini PJ (1999). Vascular endothelial growth factor (VEGF)-mediated angiogenesis is associated with enhanced endothelial cell survival and induction of Bcl-2 expression. Am J Pathol 154:375-384.

Nör JE, Mitra RS, Castle V, Mooney DJ, and Polverini PJ (2000). Thrombospondin-1 Inhibits Angiogenesis by Activating Caspase-3 and Inducing Endothelial Cell Apoptosis. J Vasc Res 37:209-218.

Polverini PJ (1996). Cellular adhesion molecules. Newly identified mediators of angiogenesis. Am J Pathol 148:10231029.

Putnam AJ and Mooney DJ (1996). Tissue engineering using synthetic extracellular matrices. Nat Med 2:824-826.

Schechner JS, Nath AK, Zheng L, Kluger MS, Hughes CC, Sierra-Honigmann MR, Lorber MI, Tellides G, Kashgarian M, Bothwell AL, and Pober JS (2000). In vivo formation of complex microvessels lined by human endothelial cells in an immunodeficient mouse. Proc Natl Acad Sci USA 97:91919196.
Strömblad S and Cheresh DA (1996). Cell adhesion and angiogenesis. Trends Cell Biol 6:462-468.

Swerlick RA, Lee KH, Li LJ, Sepp NT, Caughman SW, and Lawley TJ (1992). Regulation of vascular cell adhesion molecule 1 on human dermal microvascular endothelial cells. J Immunol 149:698-705.

Vermeulen PB, Gasparini G, Fox SB, Toi M, Martin L, McCulloch P, Pezzella F, Viale G, Weidner N, Harris AL, and Dirix LY (1996). Quantification of angiogenesis in solid human tumours: An international consensus on the methodology and criteria of evaluation. Eur J Cancer 32A:2474-2484. 